\title{
CONGENITAL ABSENCE OF THE RIGHT BRANCH OF THE BUNDLE OF HIS
}

\author{
BY
}

\author{
J. B. COAKLEY \\ From the Mater Misericordia Hospital and the Department of Anatomy, University College, Dublin \\ Received March 31, 1950
}

While congenital complete heart block is not very rare, developmental defects of bundle branches are exceptional. In the case here presented the clinical features of congenital heart disease and intraventricular block were found at necropsy to be associated with a congenital absence of the right branch, and it is therefore considered worthy of record.

\section{CASB REPORT}

A boy, aged 19 years, was admitted by Professor Henry Moore to the Mater Misericordiæ Hospital, Dublin, on May 8, 1948. Except that he had measles at 10 years of age his previous history was good but his grandmother thought that his face was "inclined to be blueish" when he was six years old; however, his school doctors passed him as fit then and subsequently at 7, 8, and 9 years of age. Nevertheless, he never played games at school. At the age of seventeen he complained that "he was not like other boys of his age, and never seemed to have energy" and that he himself noticed a blue colour of his face. Subsequently, on several occasions he "felt weak" and in one attack sank to the floor for a few seconds but did not lose consciousness. He never complained of tachycardia or dyspnœa. When first seen by us a simple exercise test produced obvious dyspnœa. His face, hands and feet were cyanotic. The heart was conspicuously enlarged to the left and right; there was a slight mitral systolic murmur; the first mitral sound was split (suggesting intraventricular heart block); the aortic and pulmonic second sounds were very faint; the pulse was feeble at the wrist and at rest was 84 a minute. The arterial blood pressure was 104/90. The respiratory rate at rest was 20; the breath sounds at the base of the right lung were diminished and there were crepitations at both lung bases. The urine showed a trace of albumin with an occasional erythrocyte.

A teleradiogram (Fig. 1) showed marked cardiac enlargement, involving especially the right atrium; the ventricular contractions were of diminished amplitude; the inferior vena cava and the pulmonary artery were slightly prominent and the aorta was scarcely discernable; the lungs were radiologically normal and the $\mathrm{X}$-ray diagnosis was congenital heart disease.

The electrocardiogram was of an unusual type (Fig. 2). At first it was thought that it might represent the concordant type of left bundle branch block, but after consideration it was concluded that it indicated an indeterminate form of intraventricular block, with probably lesions in both branches of the bundle of His. Unfortunately, the boy died suddenly, before further unipolar leads had been recorded.

\section{Post-Mortem Examination}

An examination of the thorax only was permitted. The heart was fixed in 10 per cent formalin. Sections of the atrioventricular node, bundle, and branches, from other hearts which had previously been prepared by Professor Keenan were used to confirm the findings in this heart. The 


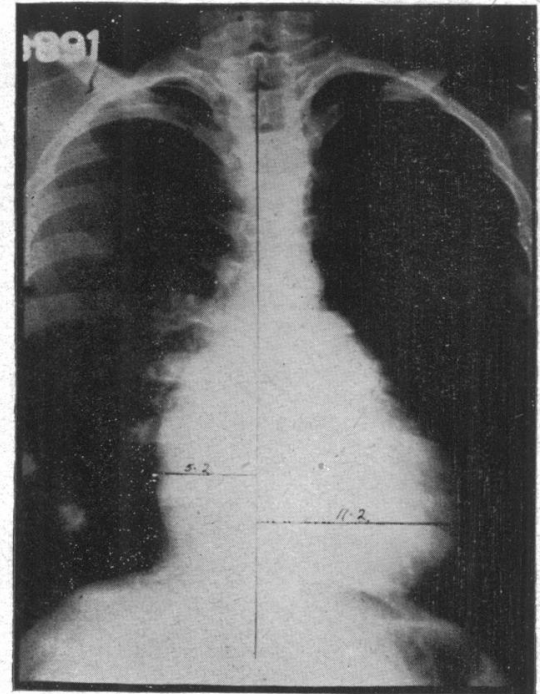

FIG. 1.-Teleradiogram (Dr. J. Geraghty). Measurements: M.R., $5 \cdot 2 \mathrm{~cm}$.; M.L., $11.2 \mathrm{~cm}$.; M.R. +M.L., $16.4 \mathrm{~cm}$. The diameter of the aortic arch could not be defined.

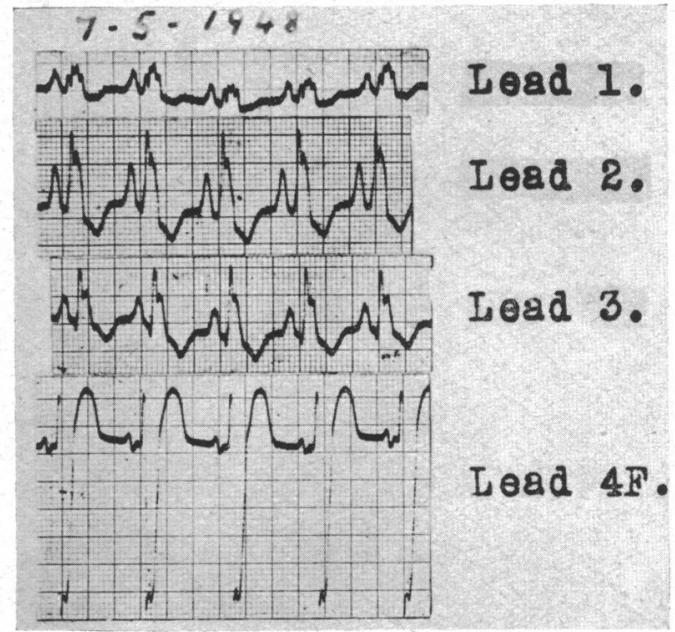

FIG. 2.-Electrocardiogram taken on 7-5-48. Sharp $P$ wave in standard leads, especially in II, and the P-R interval was shortened. QRS shows widening and slurring in all leads. S-T segment abnormal throughout. Appearances suggested indeterminate form of intraventricular block with lesions probably of both bundle branches.

method of serial section of blocks of tissue embedded in paraffin was used throughout. Al sections were cut at a thickness of $10 \mu$ and every section was mounted. The sections were stained with a modification of Masson's trichrome stain, using light green.

The Pathological and Anatomical Findings. The heart as a whole was much enlarged and together with the roots of the great vessels weighed 754 grammes. The cavity of the right ventricle was considerably smaller than normal. All the muscle walls were greatly hypertrophied. The aorta was hypoplastic, its diameter at the root of the vessel being $16.5 \mathrm{~mm}$. Dissection of the coronary arteries and their main branches revealed no evidence of occlusion. On section, the right and left coronary arteries and the interventricular branch of the left coronary showed a considerable degree of atherosclerosis, especially the interventricular branch of the left coronary artery.

Microscopic examination of the musculature of the heart revealed a considerable amount of fibrous tissue. This was present particularly between the muscle bundles but also as a replacement fibrosis.

The Conducting System. The sinu-atrial node was sectioned in its entirety and was found to be perfectly normal. The atrioventricular node was also sectioned in its entirety. While not as clearly defined as the sinu-atrial node it was recognized readily enough by its position and the characters of its fibres, and appeared to be perfectly normal.

As the node was traced forwards and to the left, many of the fibres in that portion of it nearest the atrioventricular ring commenced to penetrate the ring (Fig. 3). They constituted the atrioventricular bundle (bundle of His). As it crossed the ring the bundle was embedded in collagenous tissue. Its right border was perfectly clean cut and throughout its course a space, probably an artefact, was observed between that border and the adjacent collagenous tissue (Fig. 3 and 4). All of the conducting fibres in the bundle were cut transversely or slightly obliquely. Those on the right margin of the bundle appeared perfectly healthy and in no way differed from the fibres in the remainder of the bundle. No fibres or portions of fibres emerged from the bundle on its right side.

On reaching the summit of the muscular interventricular septum, the bundle did not divide into right and left branches as occurs in a normal heart. Instead the entire bundle mass was continued into the left branch, passing between the endocardium of the left ventricle and the muscle of the 


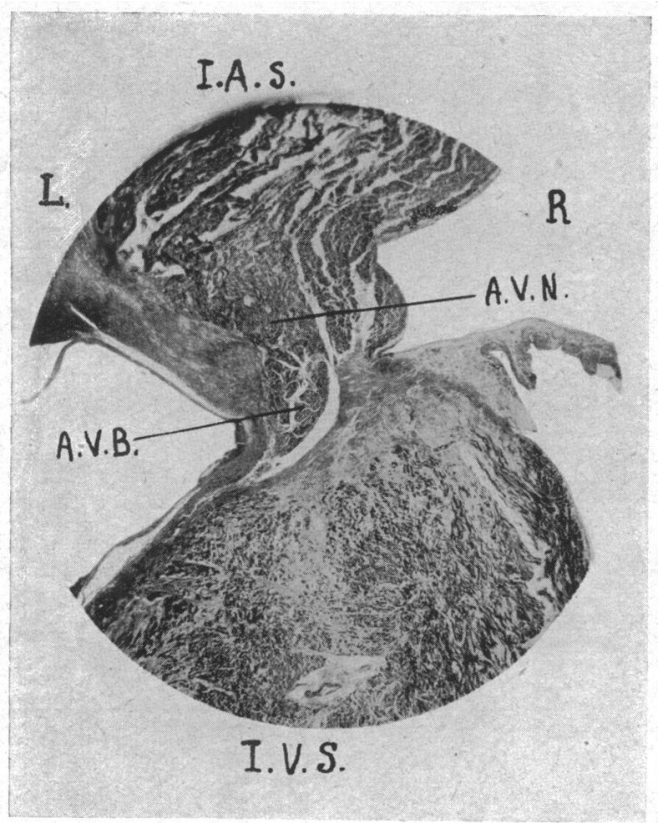

FIG. 3.-Section of the atrioventricular node and proximal portion of the bundle of His. The latter is penetrating the atrioventricular ring. I.A.S., interatrial septum; I.V.S., interventricular septum; A.V.N., atrioventricular node; A.V.B., atrioventricular bundle. Magnification,

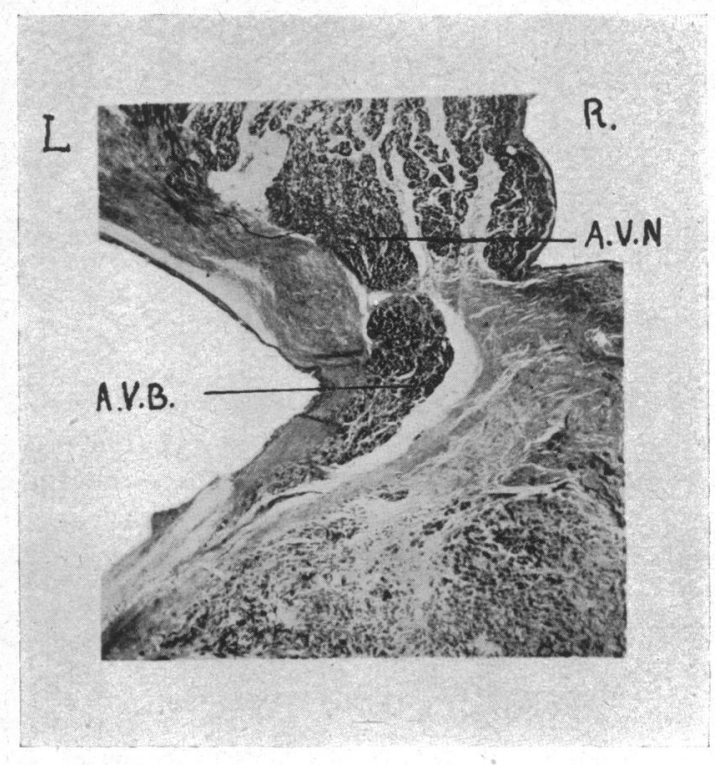

Fig. 4.-Section further forward than in Fig. 3. Showing the atrioventricular node (A.V.N.) and atrioventricular bundle (A.V.B.). Magnification, $\times 24$.

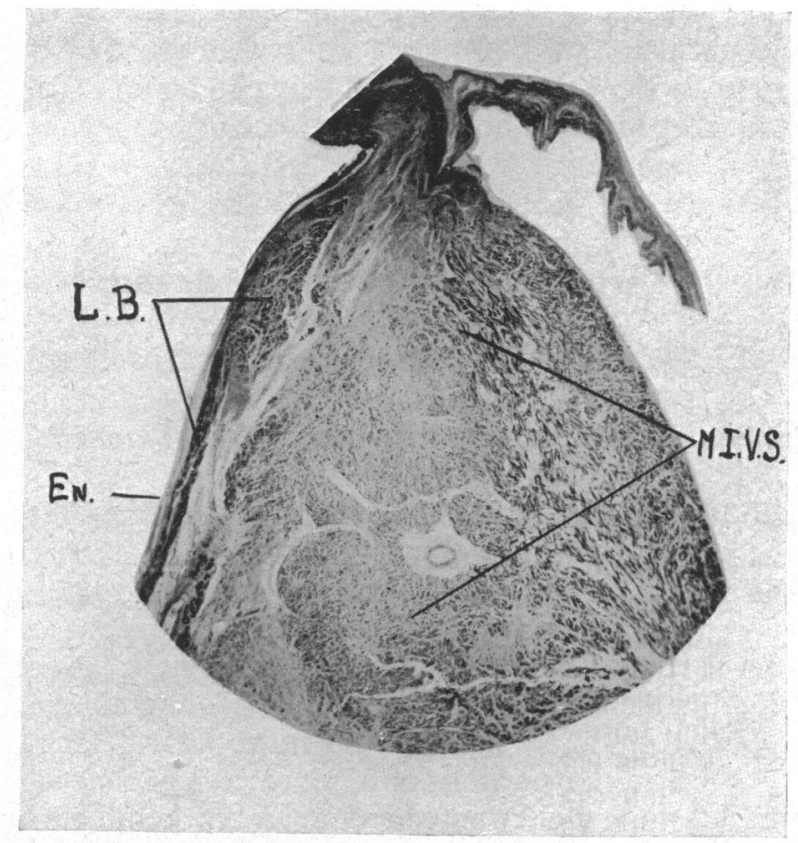

Fig. 5.-Section further forward than in Fig. 4, showing longitudinally cut fibres of the left branch (L.B.) passing towards the apex of the heart and lying directly under the endocardium of the left ventricle (En.). M.I.V.S., muscular interventricular septum. Magnification, $\times 8$. 
interventricular septum. A number of fibres emerged from this left branch, one or two at a time, and immediately joined with the muscle of the septum on its left side whence they could not be traced any further. The remaining fibres of the branch passed towards the apex of the heart, directly beneath the endocardium of the left ventricle (Fig. 5). These fibres formed a broad, flattened band, which became condensed into four main divisions. A few fibres passed from these four divisions and immediately joined with the muscle of the septum whence they could not be traced any further.

Approximately 1-1 $\frac{1}{2}$ inches below the summit of the muscular interventricular septum the anterior three of these divisions showed fibrous lesions. Here the conducting fibres were stained more palely than the adjacent muscle of the septum; their cell outlines were indistinct and the protoplasm was beaded in appearance. In these three divisions the conducting tissue was replaced by fibrous tissue and the continuity completely interrupted. These fibrous lesions were probably ischæmic in origin. Below the lesions, conducting fibres were again present and they were traced towards the apex of the ventricle. Each of the three divisions was interrupted by a second fibrous lesion. No attempt was made to trace them any further.

The fourth and most posterior division of the left branch of the bundle of His gave a branch that formed a trabeculum in the cavity of the ventricle, a portion of which unfortunately was destroyed when blood clot was being removed from the ventricle. Shortly after, this division was also completely interrupted by a fibrous lesion. Conducting fibres were present below the lesion and they were traced to the apex of the ventricle, where they were distributed to the adjacent heart muscle. In the lower portion of its course this division formed a strand which lay free in the cavity of the ventricle.

Thus all the fibres of the bundle of His that had crossed the ring from the atrioventricular node had run into the left branch. No right branch was present in this heart nor were there any conducting fibres going to the muscle of the septum on its right side.

\section{Discussion}

The possibility of a lesion in the right branch at the bifurcation of the bundle was considered. Had there been such a lesion, we think that at least a few damaged fibres would have been present to indicate the position of a right branch. No such fibres were present and there was no evidence in the sections to indicate a lesion. Further, the pathway that the right branch normally follows was sectioned down to and including a portion of the moderator band. The absence of the right branch in that pathway, in this heart, was supported by demonstration of its existence in similar sections prepared from other hearts. Sections from another heart, reported by Moore, Keenan, and Moriarty (1937) revealed a lesion in the right branch and below this lesion conducting fibres were again present in an apparently healthy condition. Similarly, in the heart now under consideration, healthy conducting fibres were observed below the lesions in the left branch; had there been a lesion high in the right branch, then healthy fibres would be expected below it, in the normal pathway of the branch. No such fibres were present in this heart.

A lesion in the right branch, at the bifurcation of the bundle, many years before the patient's death, might possibly produce the condition found in this heart. This would necessitate the complete obliteration of all right branch fibres in the region of the lesion. It would also necessitate that any healthy fibres, distal to the lesion, would have been widely separated from the bifurcation of the bundle by the enlargement of the heart. It is felt that even with such a lesion some remnants of fibres would still be present, certainly at the bifurcation of the bundle. It is very doubtful if these circumstances could result in the perfectly clear cut and healthy right border of the bundle that was found in this heart. Further, there should be some, and possibly a considerable amount of, fibrous tissue in the normal pathway of the right branch. In fact, this portion of the septum was remarkably free of fibrous tissue (Fig. 5). Such a lesion, with the sequelæ mentioned, is considered extremely unlikely to have been present in this heart. 
Examination of the atrioventricular node and the regions beyond each of its ends revealed no conducting fibres crossing the ring independent of the bundle of His. Further, no bundle of Kent was found in this heart, though a very thorough search was made for fibres crossing the atrioventricular ring from the right atrium to the right ventricle. Almost the complete ring was sectioned in this portion of the heart.

We conclude, therefore, that the absence of the right branch of the bundle in this heart must be due to a congenital defect. The work of Walls (1947) may serve to explain the congenital absence of the right branch in this heart, combined with a developmentally normal bundle and left branch; he states that the atrioventricular node represents part of the wall of the atrioventricular canal and that the bundle of His and its branches are a new formation, which arises by a process of active growth from the primitive nodal tissue. Theoretically, then, any disturbance of this growth could result in the absence of either or both of the branches. Indeed, it seems feasible that the bundle itself might fail to grow. Walls also states that the bundle and the commencement of the left branch are present at an earlier stage in development than the right. Consequently they could be perfectly normal structurally in the adult heart, even if the right branch had failed to develop. Further, the downward growth of the bundle and its branches, combined with the upward growth of the summit of the interventricular septum, could facilitate the failure of development of a branch by isolating the downwardly growing structure to one side.

I am indebted to Professor Henry Moore, at whose instigation the work was carried out, for helpful advice and assistance, and also to Professor Edward Keenan for the same reasons and for allowing the work to be done in the Anatomy Department of University College. My thanks are due to Dr. M. D. Hickey for his generous advice on the pathological features of the heart. I am also indebted to the Medical Research Council of Ireland for a grant which enabled the work to be carried out; to Dr. S. O'Carroll of Dromhair for information as to the history of the case; and to Mr. R. Kernan and Mr. P. Fletcher for valuable technical assistance.

\section{REFERENCES}

Moore, H., Keenan, E., and Moriarty, M. A. (1937). Proceedings of the Association of Physicians of Great Britain and Ireland. Quart. J. Med., 6, 475.

Walls, E. W. (1947). J. Anat., Lond., 81, 93. 